\title{
The Need to Autonomous Radiosounding System
}

\author{
Ahmed Saad Abd-Elnaby \\ The Egyptian Meteorological Authority, Koubry El-Quobba, Cairo 11784, Egypt
}

\begin{abstract}
In recent years, the upper air radiosounding system based on GPS (global positioning system) is used as an effective method resulting to improving observation accuracy and helping in simplification of the ground equipment. GPS is used to calculate the pressure and wind speed and direction. The NAVSTAR GPS has been developed by the Department of Defense of the United States, and the main purpose is to provide the military forces of the USA and its allies with a means to accurately determine their position, velocity and time, anywhere on the earth, at any time. Europe is now developing its own GPS, known as Galileo, which is independent of the USA GPS. As a result, many countries now are developing their own GPS system like Russia (GLONASS), China (Compass), India, and Japan. As a direct result, we can face the fact that each and every country will have its own GPS system and finally we come up with a crowded space. The INS (inertial navigation system) is a system that, once initialized (by acquiring the initial position, velocity, and orientation information), becomes an autonomous navigation system providing 3-D position, velocity, and attitude information. So upper air observation can take advantage of this emerging technology by using this stand alone system (INS) in measuring wind speed and direction as well as measuring the altitude we can calculate the pressure.
\end{abstract}

Key words: Global positioning system, inertial navigation system, upper air observation.

\section{Introduction}

Inertial sensor, also known as the IMU (inertial measurement unit), is a device consisting of accelerometers, gyroscopes, other electronics components, and a computer. When mounted on a moving object, the accelerometers measure the object's acceleration plus the gravitational force, while the gyroscopes provide information on the orientation of the inertial platform. These sets of information are accumulated by the sensor's microcontroller - the internal brain of the system-to produce the velocity and the position information. In addition to being a relatively environment-independent system, an inertial system provides accuracy as high as that of GPS (Global Positioning System) for the short period of time following the initialization. Moreover, inertial systems provide very high update rates compared with GPS. An accelerometer measures specific force and a gyroscope measures angular rate, both without an

Corresponding author: Ahmed Saad Abd-Elnaby, bachelor, research field: meteorological instruments. E-mail: ahmedhamada44@gmail.com. external reference. Devices that measure the velocity, acceleration, or angular rate of a body with respect to features in the environment are not inertial sensors. Most types of accelerometers measure specific force along a single sensitive axis. Similarly, most types of gyros measure angular rate about a single axis. An IMU combines multiple accelerometers and gyros, usually three of each, to produce a three-dimensional measurement of specific force and angular rate. An IMU is the sensor for an inertial navigation system, which produces an independent three-dimensional navigation solution. A major drawback of the inertial system, however, is that it suffers from drift if left unaided for a long period of time. In particular, the performance of the gyroscopes limits the overall performance of the inertial system.

Current inertial sensor development is focused on MEMS (micro-electromechanical systems) technology. This enables quartz and silicon sensors to be mass produced at low cost using etching techniques with several sensors on a single silicon wafer. MEMS sensors are small, light, and exhibit much greater shock tolerance than conventional mechanical designs. 


\section{Accelerometers}

Acceleration is a dynamic characteristic of an object, because, according to Newton's second law, it essentially requires application of a force. In effect, the position, velocity, and acceleration are all related: Velocity is a first derivative of position and acceleration is the second derivative. However, in a noisy environment, taking derivatives may result in extremely high errors, even if complex and sophisticated signal conditioning circuits are employed. Therefore, velocity and acceleration are not derived from the position detectors, but rather measured by special sensors. Most types of accelerometers measure specific force along a single sensitive axis.

Fig. 1 shows a simple accelerometer. A proof mass is free to move with respect to the accelerometer case along the accelerometer's sensitive axis, restrained by springs.

A pickoff measures the position of the mass with respect to the case. When an accelerating force along the sensitive axis is applied to the case, the proof mass will initially continue at its previous velocity, so the case will move with respect to the mass, compressing one spring and stretching the other. Stretching and compressing the springs alters the forces they transmit to the proof mass from the case. Consequently, the case will move with respect to the mass until the acceleration of the mass due to the asymmetric forces exerted by the springs matches the acceleration of the case due to the externally applied force. The resultant position of the mass with respect to the case is proportional to the acceleration applied to the case. By measuring this with a pickoff, an acceleration measurement is obtained. The exception to this is acceleration due to the gravitational force. Gravitation acts on the proof mass directly, not via the springs, and applies the same acceleration to all components of the accelerometer, so there is no relative motion of the mass with respect to the case. Therefore, all accelerometers sense specific force, the non-gravitational acceleration, not the total acceleration. The object frame for accelerometer measurements is the accelerometer case, while the reference frame is inertial space, and measurements are resolved along the sensitive axes of the accelerometers. The proof mass needs to be supported in the axes perpendicular to the sensitive axis, and damping is needed to limit oscillation of the proof mass. However, all accelerometer designs are based on the basic principle shown. Practical accelerometers used in strapdown navigation systems follow either a pendulous or vibrating-beam design, both of which are discussed later. Pendulous designs have been around for decades, while vibrating-beam accelerometers originated in the 1980s. Both types of accelerometer may be built using either conventional mechanical construction or MEMS technology. MEMS accelerometers of either design may be built with sensitive axes, both in the plane of the device and perpendicular to that plane, enabling a three-axis accelerometer triad and associated electronics to be etched onto a single silicon chip. A third type of
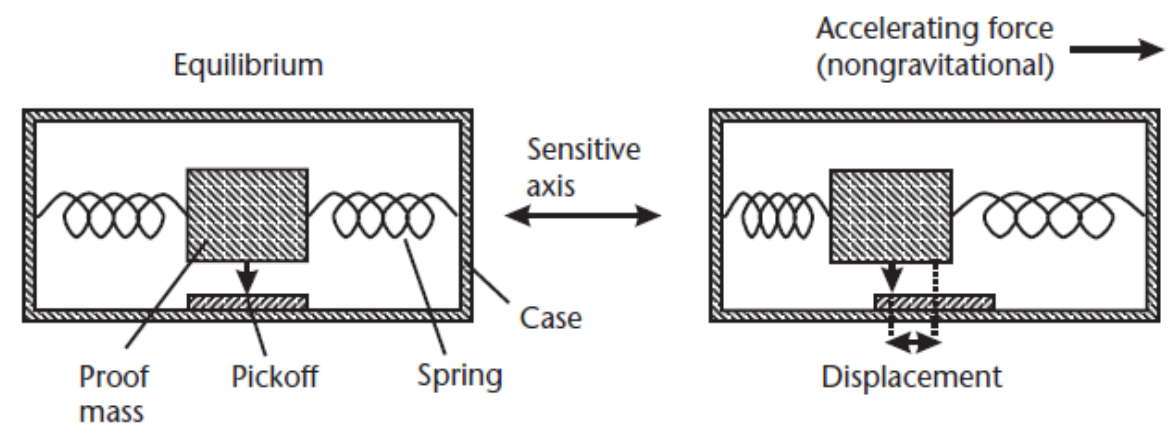

Fig. 1 A simple accelerometer. 
accelerometer, the PIGA (pendulous integrating gyro accelerometer), is only suitable for use in a platform INS (inertial navigation system) but can exhibit very high precision. In addition, research has been conducted into a number of novel accelerometer designs making use of optical, MEMS, and even atom interferometry techniques.

\subsection{Pendulous Accelerometers}

Pendulous accelerometer is a mechanical open-loop accelerometer. The proof mass is attached to the case via a pendulous arm and hinge, forming a pendulum. This leaves the proof mass free to move along the sensitive axis while supporting it in the other two axes. A pair of springs or a single spring is used to transmit force from the case to the pendulum along the sensitive axis while the hinge provides damping. Further damping may be obtained by filling the case with oil.

Although the open-loop design produces a practical accelerometer, its performance is severely limited. To resolve these problems, precision accelerometers use a closed-loop, or force feedback, configuration. In a force-feedback accelerometer, a torquer is used to maintain the pendulous arm at a constant position with respect to the case, regardless of the specific force to which the accelerometer is subject.

Both open-loop and closed-loop pendulous MEMS accelerometers are available, with the latter using an electrostatic rather than magnetic torquer. The pickoff

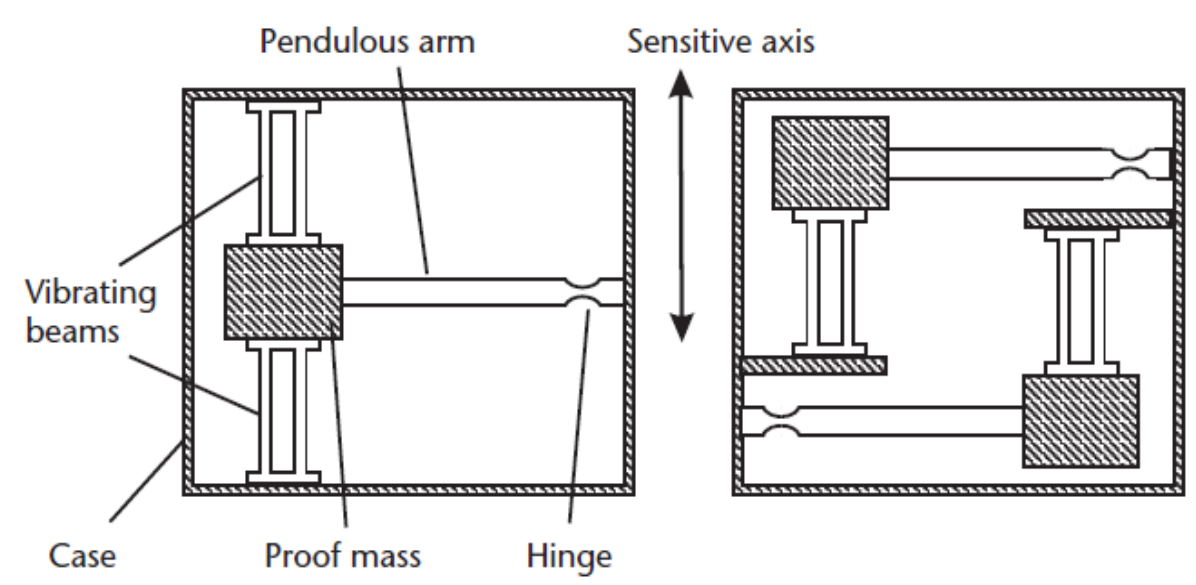

may be capacitive, as described earlier, or a resistive element mounted on the hinge, whose resistance varies as it is stretched and compressed.

\subsection{Vibrating-Beam Accelerometers}

The VBA (vibrating-beam accelerometer) or resonant accelerometer retains the proof mass and pendulous arm from the pendulous accelerometer. However, the proof mass is supported along the sensitive axis by a vibrating beam, largely constraining its motion with respect to the case. When a force is applied to the accelerometer case along the sensitive axis, the beam pushes or pulls the proof mass, causing the beam to be compressed in the former case and stretched in the latter. The beam is driven to vibrate at its resonant frequency by the accelerometer electronics. However, compressing the beam decreases the resonant frequency, whereas tensing it increases the frequency. Therefore, by measuring the resonant frequency, the specific force along the sensitive axis can be determined. Performance is improved by using a pair of vibrating beams, arranged such that one is compressed while the other is stretched. They may support either a single proof mass or two separates masses; both arrangements are shown in Fig. 2. Two-element tuning fork resonators are shown, as these are more balanced than single-element resonators. Larger scale VBAs all use quartz elements, as these provide a sharp resonance peak. 
MEMS VBAs have been fabricated out of both quartz and silicon. The VBA is an inherently open-loop device. However, the proof mass is essentially fixed; there is no variation in the sensitive axis with respect to the casing.

\section{Gyroscopes}

The definition of a gyroscope (gyro) was originally restricted to the spinning-mass type, but now encompasses all angular-rate sensors that do not require an external reference. Jean Bernard Leon Foucault is credited with inventing the spinning-mass gyro in 1852, while Charles Stark Draper led the development of high-performance gyros of this type in the 1950s. There are two main types of optical gyros. The RLG originated in the $1960 \mathrm{~s}$ as a high-performance technology, while the IFOG was developed in the 1970s as a lower cost solution. Now, the performance ranges of the RLG and IFOG overlap. A RFOG (resonant fiber-optic gyro) and a MOG (micro-optic gyro) have also been developed. Practical vibratory gyros were developed from the 1980s. All MEMS gyros operate on the vibratory principle, but larger vibratory gyros are also available and the technique spans the full performance range. A number of other gyroscope technologies, including nuclear magnetic resonance, fluoric sensors, angular accelerometers, and atom interferometry techniques, have also been researched. The object frame for gyro measurements is the gyro case, while the reference frame is inertial space, and measurements are resolved along the sensitive axis of the gyros. Thus, an IMU containing a gyro triad measures the angular rate of the IMU body with respect to inertial space in body axes, the vector.

\subsection{Spinning-Mass Gyroscopes}

Spinning-mass gyros operate on the principle of conservation of angular momentum. Part of Newton's second law of dynamics states that the angular momentum of a body with respect to inertial space will remain unchanged unless acted upon by a torque (force $\times$ distance). Therefore, if a spinning mass is mounted in an instrument case such that it is free to rotate about both of the axes perpendicular to its spin axis, it will remain aligned with respect to inertial space as the case is rotated. Pickoffs that measure the orientation of the spinning mass with respect to the case thus provide measurements of the instrument case's attitude about two axes. Such a device is known as a gyrocompass and is used as a stand-alone attitude sensor on many ships and aircraft. However, the resolution of attitude pickoffs limits the usefulness of gyrocompasses for inertial navigation.

\subsection{Optical Gyroscopes}

Optical gyroscopes work on the principle that, in a given medium, light travels at a constant speed in an inertial frame. If light is sent in both directions around a non rotating closed-loop waveguide made of mirrors or optical fiber, the path length is the same for both beams. However, if the waveguide is rotated about an axis perpendicular to its plane, then, from the perspective of an inertial frame, the reflecting surfaces are moving further apart for light traveling in the same direction as the rotation and closer together for light traveling in the opposite direction. Thus, rotating the waveguide in the same direction as the light path increases the path length and rotating it in the opposite direction decreases the path length. By measuring the changes in path length, the angular rate of the waveguide with respect to inertial space can be determined. Note that, from the perspective of the rotating frame, the path length remains unchanged but the speed of light changes.

\subsection{Vibratory Gyroscopes}

A vibratory gyroscope comprises an element that is driven to undergo simple harmonic motion. The vibrating element may be a string, beam, pair of beams, tuning fork, ring, cylinder, or hemisphere. All operate on the same principle, which is to detect the 


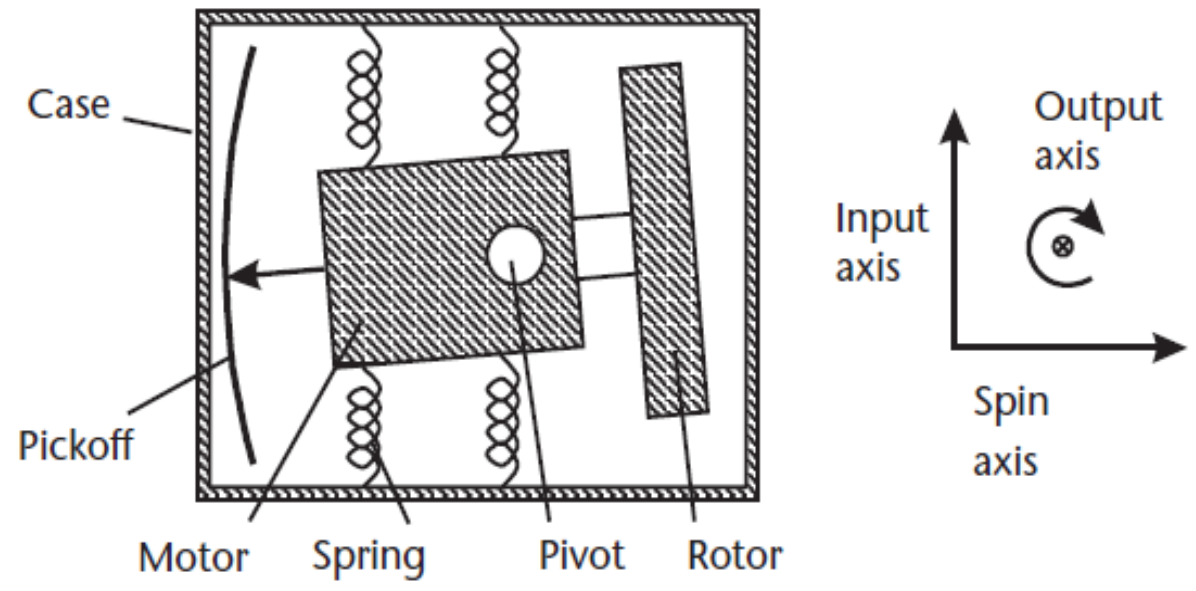

Fig. 3 Open-loop single-degree-of freedom spinning-mass gyro.

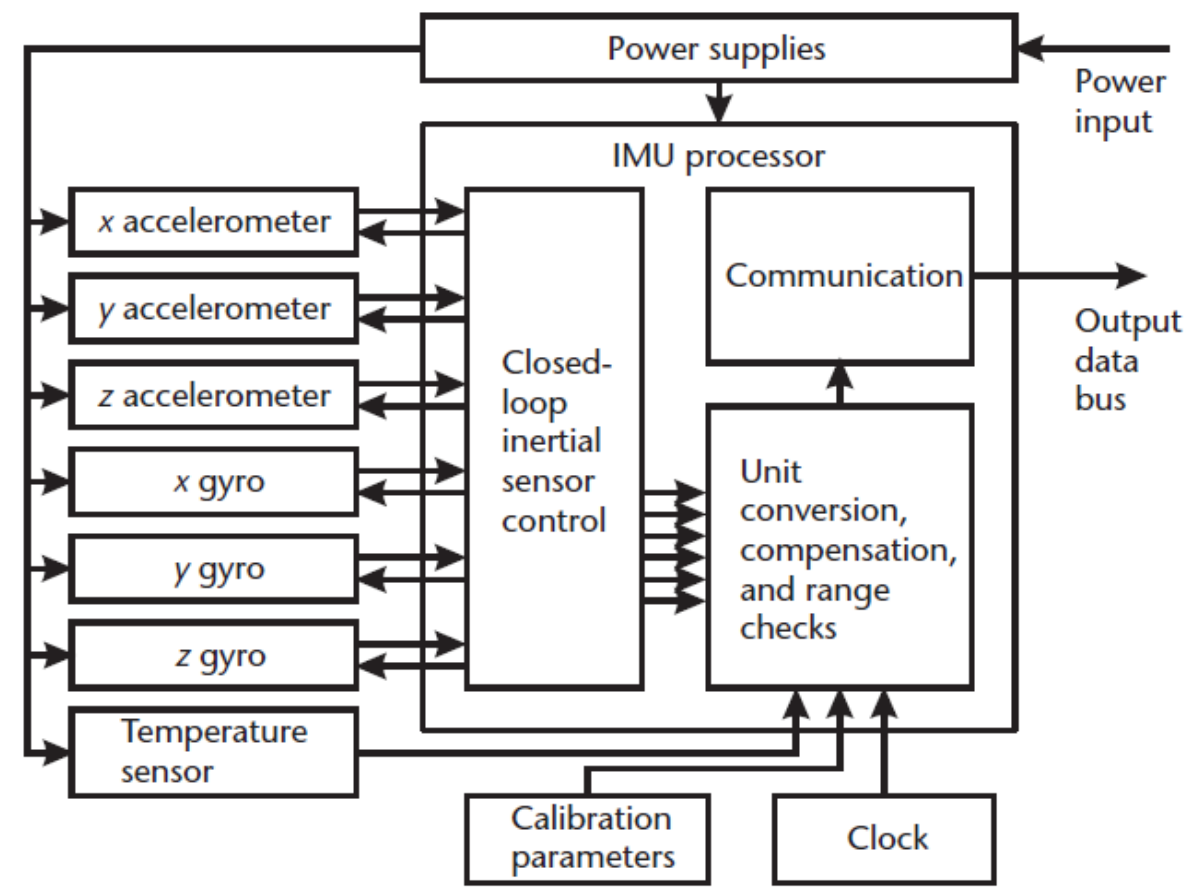

Fig. 4 Schematic of an inertial measurement unit.

Coriolis acceleration of the vibrating element when the gyro is rotated. This is easiest to illustrate with a vibrating string.

Most vibratory gyros are low-cost, low-performance devices, often using MEMS technology and with quartz giving better performance than silicon. The exception is the HRG (hemispherical resonator gyro), which can offer aviation grade performance. The HRG is light, compact, and operates in a vacuum, so has become popular for space applications.

\section{Inertial Measurement Units}

Fig. 4 shows the main elements of a typical inertial measurement unit: accelerometers and gyroscopes, the IMU processor, a calibration-parameters store, a temperature sensor, and associated power supplies. Most IMUs have three accelerometers and three single-degree-of-freedom gyroscopes, mounted with orthogonal sensitive axes. However, some IMUs incorporate additional inertial sensors in a skewed configuration to protect against single sensor failure. 
The IMU processor performs unit conversion on the inertial sensor outputs, provides compensation for the known errors of the inertial sensors, and performs range checks to detect sensor failure. It may also incorporate closed-loop force feedback or rebalance control for the accelerometers and/or gyros. Unit conversion transforms the inertial sensor outputs from potential difference, current, or pulses into units of specific force and angular rate.

\section{Conclusions}

The importance of upper air observations in weather forecasting motivates the metrological community and forces them to find new and advanced technologies in this field. The GPS radiosounding fulfills the needs of upper air observations. The developed countries adopted this technology and launched their own GPS systems that open the race for other countries to do the same which leads to a crowded space. The developing and least developing countries will be left behind in this unfair race. So we attempt to encourage people who are working in the meteorology and climate and the manufacturing firms to understand the theory and physics behind the INS as an alternative technology. The introduction of new technology should be as and when proven and must be consistent with existing system and supporting structure. The INS is capable of extracting the same information as from the GPS. This information consists of wind speed and direction and altitude to calculate pressure.

\section{References}

[1] Paul, D. G. 2008. Principles of GNSS, Inertial, and Multisensor Integrated Navigation Systems. Boston: Artech House.

[2] Lawrence, A., ed. 2001. Modern Inertial Technology. New York, NY: Springer-Verlag.

[3] Norgia, M., and Donati, S. 2001. "Hybrid Opto-Mechanical Gyroscope with Injection-Interferometer Readout.” Electronics Letters 37 (12): 756-8.

[4] El-Sheimy, N., and Niu, X. "The Promise of MEMS to the Navigation Community.” Inside GNSS, March-April 2007.

[5] Barbour, N. M. 2003. "Inertial Navigation Sensors." Advances in Navigation Sensors and Integration Technology, NATO RTO Lecture Series-232, London, UK.

[6] Jekeli, C. 2004. "Cold Atom Interferometer as Inertial Measurement Unit for Precision Navigation.” Proc. ION 60th AM, Dayton, OH.

[7] Sorg, H. W. 1976. "From Serson to Draper-Two Centuries of Gyroscopic Development.” Navigation: JION 23 (4).

[8] El-Rabbany, A., and El-Diasty, M. 2004. "An Efficient Neural Network Model for De-noising of MEMS-Based Inertial Data.” Journal of Navigation 57 (3). 